International Journal of Modern Physics A, (C) World Scientific Publishing Company

\title{
NEUTRINO PHYSICS WITH SMALL EXTRA DIMENSIONS
}

\author{
MATTHIAS NEUBERT \\ Newman Laboratory of Nuclear Studies, Cornell University \\ Ithaca, New York 14853, U.S.A.
}

\begin{abstract}
We study neutrino physics in the context of the localized gravity model with nonfactorizable metric proposed by Randall and Sundrum. Identifying the right-handed neutrino with a bulk fermion zero mode, which can be localized on the "hidden" 3-brane in the Randall-Sundrum model, we obtain naturally small Dirac neutrino masses without invoking a see-saw mechanism. Our model predicts a strong hierarchy of neutrino masses and generically large mixing angles.
\end{abstract}

\section{Introduction}

Theories with extra spatial dimensions have received great attention recently, when it was shown that they could provide a solution to the gauge-hierarchy problem. If space-time is a product of Minkowski space with $n$ compact dimensions, with Standard Model fields localized in the three extended spatial dimensions (i.e., on a 3-brane) and gravity propagating in the extra space, then the strength of gravity on the 3-brane is governed by an effective Planck scale $M_{\mathrm{Pl}}^{2}=M^{n+2} V_{n}$, where $M$ is the fundamental scale of gravity and $V_{n}$ the volume of the compact space. If this space is sufficiently large, the fundamental scale $M$ can be of order $1 \mathrm{TeV}$, thus removing the large disparity between the gravitational and the electroweak scales.

An intriguing alternative to the above scenario invokes a non-factorizable geometry with a metric that depends on the coordinates of the extra dimensions In the simplest scenario due to Randall and Sundrum (RS) one considers a single extra dimension, taken to be a $S^{1} / Z_{2}$ orbifold parameterized by a coordinate $y=r_{c} \phi$, with $r_{c}$ the radius of the compact dimension, $-\pi \leq \phi \leq \pi$, and the points $(x, \phi)$ and $(x,-\phi)$ identified. There are two 3-branes located at the orbifold fixed points: a "visible" brane at $\phi=\pi$ containing the Standard Model fields, and a "hidden" brane at $\phi=0$. The solution of Einstein's equations for this geometry (with carefully tuned brane tensions) leads to the non-factorizable metric $d s^{2}=e^{-2 k r_{c}|\phi|} \eta_{\mu \nu} d x^{\mu} d x^{\nu}-r_{c}^{2} d \phi^{2}$, where $x^{\mu}$ are the coordinates on the fourdimensional surfaces of constant $\phi$, and the curvature parameter $k$ is of order the fundamental Planck scale $M$. In between the two branes is a slice of $\mathrm{AdS}_{5}$ space.

With this setup, the effective Planck scale seen by particles confined to fourdimensional space-time is of order the fundamental scale $M$. However, the "warp factor" $e^{-2 k r_{c}|\phi|}$ in the metric has important implications for the masses of particles confined to the visible brane. After field renormalization any mass parameter $m_{0}$ in the fundamental theory is promoted into an effective mass parameter $m=e^{-k r_{c} \pi} m_{0}$ 
governing the physical properties of particles on the brane. With $k r_{c} \approx 12$ this mechanism produces weak-scale physical masses and couplings from fundamental masses and couplings of order the Planck scale. As a consequence of the warp factor, the Kaluza-Klein excitations of bulk fields have weak-scale mass splittings and couplings, 䀝 in contrast with the Kaluza-Klein spectra in models with large extra dimensions.

Resolving the hierarchy problem by introducing extra dimensions poses new challenges. In particular, the see-saw mechanism for generating small neutrino masses cannot be invoked if the highest energy scale governing physics on the visible brane is the weak scale. Although several four-dimensional alternatives to the seesaw mechanism not requiring a high-energy scale have been proposed, it would be interesting to find new mechanisms that are intrinsically higher dimensional. In the context of models with large extra dimensions ideas in this direction have been presented in Refs. 6 国. They contain a massless Standard Model singlet propagating in the bulk of the extra compact space, which serves as a right-handed neutrino. Then the effective four-dimensional Yukawa coupling is suppressed by a volume factor $1 / \sqrt{V_{n}}$, reflecting the small overlap between the right-handed neutrino in the bulk and the left-handed one on the 3-brane. By construction, this factor provides a suppression of neutrino masses of order $v / M_{\mathrm{Pl}}$, reminiscent of the seesaw mechanism. However, this idea does not work in a scenario with small extra dimensions, simply because of the lack of a volume suppression factor.

In a recent work we have investigated the possibility of incorporating bulk fermions in the RS model. Starting point is the action for a Dirac fermion with mass $m$ of order the fundamental scale $M$ propagating in a five-dimensional space with the RS metric. We perform the Kaluza-Klein decomposition taking into account the orbifold boundary conditions on the branes, which imply that either all left-handed or all right-handed fields must be $Z_{2}$-odd and thus vanish on the two 3 -branes. Which of these choices is realized in nature is a question that cannot be answered without understanding in detail the physics on the 3-branes. The masses of the Kaluza-Klein fermions are given by $m_{n}=e^{-k r_{c} \pi} k x_{n}$, where $x_{n}=O(1)$ are roots of some Bessel functions, and the small parameter $\epsilon \equiv e^{-k r_{c} \pi} \sim 10^{-16}$ sets the ratio between the electroweak and the gravitational scales. Hence the masses $m_{n}$ are of order the weak scale $v$. Of particular importance to our study are the zero modes supported by the RS geometry, i.e., solutions with $x_{n}=0$. The corresponding wave functions are given by

$$
f_{0}^{L, R}(\phi) \propto \sqrt{\frac{1 \pm 2 \nu}{1-\epsilon^{1 \pm 2 \nu}}} \epsilon^{\mp \nu(|\phi| / \pi-1)},
$$

where $\nu=m / k$ is a parameter of order unity. Only one of the zero modes is allowed by the orbifold symmetry. This mode exists irrespective of the value of the fermion mass $m$ in the five-dimensional theory. Note that for $\nu>\frac{1}{2}$ the right-handed zero mode has a very small wave function on the visible brane: $f_{0}^{R}(\pi) \propto \epsilon^{\nu-\frac{1}{2}}$. This property allows us to obtain small neutrino masses. 


\section{Yukawa interactions and neutrino phenomenology}

We focus first on a single fermion generation and consider a scenario where all matter and gauge fields charged under the Standard Model gauge group are confined to the visible brane at $\phi=\pi$, whereas a gauge-singlet fermion field propagates in the bulk. After integration over the compact extra dimension we obtain a tower of four-dimensional Kaluza-Klein fermions with weak-scale mass splitting. We choose boundary conditions such that there is a right-handed zero mode with wave function $f_{0}^{R}(\phi)$ given in (11). Only this choice will lead to an interesting neutrino phenomenology.

We introduce a Yukawa coupling of the bulk fermion with the Higgs and lepton fields. With our choice of boundary conditions all left-handed Kaluza-Klein modes vanish at the visible brane, so only the right-handed modes can couple to the Standard Model fields on the brane. Rescaling the Standard Model fields so as to restore a canonical normalization, and inserting for the bulk fermion the Kaluza-Klein decomposition, we find the action

$$
S_{Y}=-\sum_{n \geq 0} \int \mathrm{d}^{4} x\left\{y_{n} \bar{L}(x) \widetilde{H}(x) \psi_{n}^{R}(x)+\text { h.c. }\right\}
$$

with effective Yukawa couplings $y_{n}=Y_{5} f_{n}^{R}(\pi)$, where $Y_{5}$ is naturally of order unity. After electroweak symmetry breaking, this Yukawa interaction gives rise to a neutrino mass term $\bar{\psi}_{L}^{\nu} \mathcal{M} \psi_{R}^{\nu}+$ h.c. in the basis $\psi_{L}^{\nu}=\left(\nu_{L}, \psi_{1}^{L}, \ldots, \psi_{n}^{L}\right)$ and $\psi_{R}^{\nu}=\left(\psi_{0}^{R}, \psi_{1}^{R}, \ldots, \psi_{n}^{R}\right)$, with $n \rightarrow \infty$. As a consequence, there will be a mixing of the Standard Model neutrino $\nu_{L}$ with the heavy, sterile (with respect to the Standard Model gauge interactions) bulk neutrinos $\psi_{n}^{L}$. In order to obtain a light neutrino we need $\left|y_{0}\right| \ll 1$, which requires having a very small wave function of the zero mode on the visible brane, i.e., $\left|f_{0}^{R}(\pi)\right| \ll 1$. But this is precisely what happens if the fundamental fermion mass $m$ satisfies the condition $m>k / 2$.

In order to study the properties of the physical neutrino states we diagonalize the squared mass matrix $\mathcal{M M}^{\dagger}$. The eigenvalues of this matrix are the squares of the physical neutrino masses, and the unitary matrix $U$ defined such that $U^{\dagger} \mathcal{M} \mathcal{M}^{\dagger} U$ is diagonal determines the left-handed neutrino mass eigenstates via $\psi_{L}^{\nu}=U \psi_{L}^{\text {phys }}$. We denote by $m_{\nu}$ the mass of the lightest neutrino $\nu_{L}^{\text {phys }}$ and define a mixing angle $\theta_{\nu}$ such that $\nu_{L}=\cos \theta_{\nu} \nu_{L}^{\text {phys }}+\ldots$, where the dots represent the admixture of heavy, sterile bulk states. To leading order in the small parameter $\left|y_{0}\right| \ll 1$ we obtain (for $\nu>\frac{1}{2}$ )

$$
m_{\nu} \sim M\left(\frac{v}{M}\right)^{\nu+\frac{1}{2}}, \quad \tan ^{2} \theta_{\nu}=\frac{1}{2 \nu+1} \frac{v_{0}^{2}\left|Y_{5}\right|^{2}}{k^{2}} .
$$

The first result is remarkable, as it provides a parametric dependence of the neutrino mass on the ratio of the electroweak and Planck scales that is different from the seesaw relation $m_{\nu} \sim v^{2} / M$, except for the special case where $\nu=\frac{3}{2}$. This flexibility allows us to reproduce a wide range of neutrino masses without any fine-tuning. 
For instance, taking $v / M=10^{-16}$, the phenomenologically interesting range of $m_{\nu}$ between $10^{-5} \mathrm{eV}$ and $10 \mathrm{eV}$ can be covered by varying $\nu$ between 1.1 and 1.5 .

The measurement of the invisible width of the $Z^{0}$ boson, which yields $n_{\nu}=$ $2.985 \pm 0.008$ for the apparent number of light neutrinos, implies that the mixing angle $\theta_{\nu}$ must be of order a few percent. (Similar constraints follow from lepton universality.) For instance, assuming an equal admixture of sterile neutrinos for the three generations of light neutrinos, we obtain $n_{\nu}=3 \cos ^{2} \theta_{\nu}$ and hence $\tan ^{2} \theta_{\nu}=$ $0.005 \pm 0.003$. In the context of our model this implies that $v_{0}\left|Y_{5}\right| / k \sim 0.1$, which is possible without much fine-tuning. We emphasize, however, that it would be unnatural to have the dimensionless combination $v_{0}\left|Y_{5}\right| / k$ much less than unity, so a mixing angle $\theta_{\nu}$ not much smaller than the current experimental bound is a generic feature of our scenario, which can be tested by future precision measurements.

We now generalize our mechanism to three neutrino flavors and more than one bulk fermion. Interestingly, such a generalization is forced upon us by the requirement that the parity anomaly for fermions in an odd number of dimensions vanish. When an odd number of bulk fermions in five dimensions are coupled to a gauge field or gravity, parity invariance is broken at the quantum level. To obtain a minimal model that is anomaly free we introduce two bulk fermions, so there are two massless right-handed zero modes. In order to explain the atmospheric and solar neutrino anomalies in terms of neutrino oscillations one needs two very different mass-squared differences: $\Delta m_{21}^{2} \ll \Delta m_{32}^{2}$, where $\Delta m_{i j}=m_{\nu_{i}}^{2}-m_{\nu_{j}}^{2}$, and by convention $m_{\nu_{1}}<m_{\nu_{2}}<m_{\nu_{3}}$. This requires a minimum of two massive neutrinos; however, the third neutrino can be massless. In our minimal model this is indeed what happens.

In order to explore this minimal model in more detail we ignore, for simplicity, the heavy Kaluza-Klein excitations of the bulk fermions and focus only on the zero modes. The admixture of weak-scale sterile neutrino states must be strongly suppressed. It is natural to allow for the possibility that the two bulk fermions have different masses $m_{1}>m_{2}$ (of order the Planck scale) in the fundamental theory, and that they couple with similar strength to the three left-handed neutrino flavors. Diagonalizing the resulting neutrino mass matrix $\bar{\psi}_{L}^{\nu} \mathcal{M} \psi_{R}^{\nu}+$ h.c. in the truncated basis $\psi_{L}^{\nu}=\left(\nu_{e}^{L}, \nu_{\mu}^{L}, \nu_{\tau}^{L}\right)$ and $\psi_{R}^{\nu}=\left(\psi_{0}^{R, 1}, \psi_{0}^{R, 2}\right)$ to leading order in $\epsilon$ we find that the physical neutrino mass eigenstates comprise a massless left-handed neutrino $\nu_{1}$, a very light Dirac neutrino with mass $m_{\nu_{2}}$, and a light Dirac neutrino with mass $m_{\nu_{3}}$, where

$$
m_{\nu_{2}}^{2} \sim M^{2}\left(\frac{v}{M}\right)^{2 \nu_{1}+1}, \quad m_{\nu_{3}}^{2} \sim M^{2}\left(\frac{v}{M}\right)^{2 \nu_{2}+1}
$$

An interpretation of the solar neutrino anomaly in terms of neutrino oscillations based on the MSW effect yields values of $\Delta m_{21}^{2}$ in the range $10^{-6}-10^{-5} \mathrm{eV}^{2}$, whereas oscillations in vacuum would require a smaller value of order $10^{-10} \mathrm{eV}^{2}$. Such masses can be reproduced in our model by setting $\nu_{1} \approx 1.34-1.37$ and $\nu_{1} \approx 1.5$, respectively. An explanation of the atmospheric neutrino anomaly in terms of neutrino oscillations yields $\Delta m_{32}^{2}$ in the range $5 \cdot 10^{-4}-6 \cdot 10^{-3} \mathrm{eV}^{2}$, which we can reproduce 
by taking $\nu_{2} \approx 1.27-1.29$. In other words, we can understand the observed hierarchy of the experimentally favored neutrino masses in terms of a small difference of the bulk fermion masses in the fundamental theory.

Despite the fact that a strong neutrino mass hierarchy is a generic feature of our model, the mixing matrix $U$ relating the neutrino flavor and mass eigenstates does not contain any small parameter. Defining $\nu_{f}=\sum_{i=1}^{3} U_{f i} \nu_{i}$ we find that all the entries $U_{f i}$ are of order unity. A mixing matrix of this type, which lacks the strong hierarchy of the quark mixing matrix, is compatible with the experimental constraints on the neutrino mixing angles.

\section{Conclusions}

We have presented an intriguing scenario for the origin of neutrino masses and mixings in the context of the Randall-Sundrum extra-dimension model with nonfactorizable geometry. Bulk fermion states in this model have chiral zero modes localized on one of the two 3-branes, depending on the choice of orbifold boundary conditions. If the localization is done on the "hidden" brane, then these states have a wave function on the visible brane that is power-suppressed in the ratio of the weak scale to the fundamental Planck scale. Coupling the Higgs and left-handed lepton fields of the Standard Model, localized on the visible brane, with a bulk righthanded neutrino provides a new mechanism for obtaining small neutrino masses. Remarkably, this mechanism leads to a generalization of the see-saw formula with a different parametric dependence on the ratio $v / M$, which can easily reproduce neutrino masses in the range $10^{-5} \mathrm{eV}$ to $10 \mathrm{eV}$. With an even number of bulk fermions one can obtain viable models of neutrino flavor oscillations, which naturally predict a mass hierarchy and a neutrino mixing matrix not containing any small parameter.

\section{Acknowledgements}

It is a pleasure to thank Yuval Grossman for collaboration on the subject of this talk. This work was supported in part by the National Science Foundation.

\section{References}

1. N. Arkani-Hamed, S. Dimopoulos and G. Dvali, Phys. Lett. B 429, 263 (1998);

I. Antoniadis, N. Arkani-Hamed, S. Dimopoulos and G. Dvali, Phys. Lett. B 436, 257 (1998).

2. L. Randall and R. Sundrum, Phys. Rev. Lett. 83, 3370 (1999).

3. L. Randall and R. Sundrum, Phys. Rev. Lett. 83, 4690 (1999).

4. J. Lykken and L. Randall, JHEP 0006, 014 (2000).

5. W.D. Goldberger and M.B. Wise, Phys. Rev. D 60, 107505 (1999).

6. N. Arkani-Hamed, S. Dimopoulos, G. Dvali and J. March-Russell, Neutrino masses from large extra dimensions, preprint hep-ph/9811448.

7. N. Arkani-Hamed and M. Schmaltz, Phys. Rev. D 61, 033005 (2000).

8. G. Dvali and A. Yu. Smirnov, Nucl. Phys. B 563, 63 (1999).

9. Y. Grossman and M. Neubert, Phys. Lett. B 474, 361 (2000). 